\title{
Distribution of carbapenem resistant Acinetobacter Baumannii and Pseudomonas aeruginosa and ESBL-producing organisms colonization among intensive care patients
}

P Santanirand ${ }^{1 *}$, K Malathum $^{2}$, T Chadlane ${ }^{1}$, W Laolerd $^{1}$

From International Conference on Prevention \& Infection Control (ICPIC 2011)

Geneva, Switzerland. 29 June - 2 July 2011

\section{Introduction / objectives}

Infection with carbapenem resistant $A$. baumannii (CR$A B$ ) and $P$. aeruginosa (CR-PS) as well as ESBL-producing organisms have caused high rate of mortality. This project was aimed to survey the prevalence of drug resistant organisms colonization in critical care patients as a part of infection control program.

\section{Methods}

Perianal swab, urine (from Foley's catheter) and endotracheal catheter (ET) were collected from patients who were admitted to intensive care units of a 1000-bed hospital. Isolation of CR-AB and CR-PS was using EMB agar containing $12 \mathrm{ug} / \mathrm{ml}$ of either imipenem or meropenem. The MacConkey agar containing $1 \mathrm{ug} / \mathrm{ml}$ of cefotaxime was used for screening of ESBL-producing organisms. The MIC of these organisms was performed using THANF customised panel (Sensititre, UK).

\section{Results}

A total of 81 isolates was detected from 39 patients. There were 53, 22 and 6 isolates of ESBL-producing organisms, $C R-A B$ and CR-PS, respectively. Perianal was found to be the most common site for colonisation with ESBL-producing organisms (45/60 isolates) while 8 of 14 isolates from ET were CR- $A B$. All CR- $A B$ isolates resisted to nearly all tested antibiotics. However, all isolates were susceptible to colistin and tigecycline with the MIC90 at $\leq 1$ and $1 \mathrm{ug} / \mathrm{ml}$, respectively. In contrast, the ESBL-producing organisms remained susceptible to all tested carbapenems. Nevertheless, $68 \%$ of these isolates resisted to fluoroquinolones.

\section{Conclusion}

This project is a part of implementation of hospitalacquired infection control policy. The data demonstrated the existing of various multiple-drugs resistant organisms in critical care patients which would be a challenging task for infectious control.

\section{Disclosure of interest}

None declared.

\section{Author details}

'Pathology, Faculty of Medicine, Ramathibodi Hospital, Mahidol University, Bangkok, Thailand. 'Medicine, Faculty of Medicine, Ramathibodi Hospital, Mahidol University, Bangkok, Thailand.

Published: 29 June 2011

doi:10.1186/1753-6561-5-S6-P293

Cite this article as: Santanirand et al:: Distribution of carbapenem resistant Acinetobacter Baumannii and Pseudomonas aeruginosa and ESBL-producing organisms colonization among intensive care patients. BMC Proceedings 2011 5(Suppl 6):P293. 